\title{
EL TRANSPORTE PÚBLICO \\ DE PASAJEROS EN LA CIUDAD \\ DE SANTA FE: UN ANÁLISIS DE ACCESIBILIDAD Y VULNERABILIDAD SOCIAL SOBRE EL TERRITORIO
}

\author{
Tesista \\ María Pía Giménez Puentes
}

Directora de tesis

Silvia Regoli

La planificación del territorio debe acompañar las transformaciones sociales que se producen a lo largo del tiempo. En dichos procesos se estudia las formas en que el servicio de Transporte Público de Pasajeros — colectivosinfluye de manera sustancial en el desarrollo de las diversas actividades, que enfrentan y realizan las personas en su vida cotidiana. Así, la falta de coordinación de los servicios en el territorio puede generar exceso de demanda en algunas áreas y de oferta en otras.

Es el Estado Local quién tiene la responsabilidad en brindar una prestación continua, regular, universal y accesible para toda la población.

Actualmente, el Transporte Público de Pasajeros (TPP) de la ciudad de Santa Fe presenta una demanda del servicio condicionada, principalmente, en aquellas zonas del territorio donde se localiza la población más vulnerable en términos socioeconómicos.

Asimismo, la falta de infraestructura y la existencia de áreas vacantes a las que el servicio no llega, lo que no permite garantizar la continuidad y universalidad en su prestación. Acompañando dicha situación con un alto crecimiento del uso del automóvil particular en desmedro del transporte público. Entre los principales motivos se encuentran los altos tiempos de espera, los recorridos y las condiciones del servicio para brindar una mejor cobertura a la población, la tarifa, entre otros. Esto lleva a cuestionar las garantías de accesibilidad al TPP.

Es por ello que, las demandas sociales producto de los cambios vertiginosos que vienen afectando el desenvolvimiento inclusivo de las sociedades llevan a buscar una mejor adaptación del TPP, enfocando el servicio desde con una mirada integral que priorice los intereses de los usuarios del mismo.

La presente tesis se encuentra organizada en cinco capítulos. En el primer capítulo introductorio se encuentra la descripción de la situación problemática, las preguntas, objetivos e hipótesis de investigación. En el segundo capítulo se exponen las referencias 
bibliográficas que dan sustento a la investigación realizada. El tercer capítulo se detalla la metodología utilizada para el análisis de la situación actual del servicio de transporte público de pasajeros y su accesibilidad en zonas de vulnerabilidad social. El cuarto capítulo consta de los resultados empíricos alcanzados a través de la metodología implementada. Por último, en el quinto capítulo se expone las principales conclusiones obtenidas y se comentan las posibles futuras líneas de investigaciones a trabajar.

La tesis se propone contribuir al diseño de políticas públicas para el Transporte Urbano de Pasajeros de la ciudad de Santa Fe a partir de un diagnóstico actualizado de la oferta y la demanda, focalizando en la disponibilidad del servicio para la población vulnerable.

A través de una técnica mixta — cuantitativa y cualitativa - se buscó generar una caracterización de la población en términos socioeconómico y establecer la condición de accesibilidad por parte de la población santafesina al sistema de colectivos.

Para ello, Se seleccionaron variables relevantes para caracterizar el sistema de transporte urbano en la ciudad de Santa Fe así como las variables necesarias para determinar y evaluar la cobertura del servicio dada la realidad territorial y la densidad poblacional. Se construyeron distintos indicadores, a través de un Sistema de Información Geográfica (SIG) ${ }^{1}$ para lograr una interpretación de la información recolectada, con el objetivo de generar herramientas que permitan monitorear el comportamiento del sector de TPP. A los fines de caracterizar la condición socioeconómica de la población, desde una perspectiva estructural, se tomaron variables asociadas a la calidad habitacional, al acceso de otros bienes y servicios públicos, a la posesión de activos físicos (como variable proxy de la riqueza), nivel educativo y situación ocupacional, entre otras. Con estas variables se elaboró un índice de nivel socioeconómico (Índice SES) usando técnicas de estadística multivariada.

Los resultados empíricos muestran la existencia de una importante asociación entre el nivel socioeconómico de la población y variables relacionadas a la accesibilidad del transporte. En particular se observa una desigualdad significativa en el acceso al TPP, en detrimento de las zonas caracterizadas por un menor estatus socioeconómico.

El principal hallazgo, que permite refutar la hipótesis de investigación planteada, expone que las políticas de planificación territorial del servicio de TPP (colectivos) de la ciudad de Santa Fe no satisfacen a la población socio-económicamente vulnerable. En otras palabras, aquella población de menores recursos que se localiza en zonas de vulnerabilidad social es compatible con un escaso acceso al TPP, lo que se expresa con las grandes distancias recorridas y la menor cantidad de líneas disponibles.

En lo que respecta al servicio de TPP actualmente existen áreas vacantes en lo que hace a la prestación del servicio (con nula o baja cobertura del transporte público de colectivos). Entre los resultados empíricos alcanzados se destaca que a menor nivel socioeconómico de la población, mayores son las distancias recorridas a las líneas de TPP más próxima, destacando que las mayores diferencias se encuentran entre aquella población del menor estatus socioeconómico.

Tanto a nivel de barrios como de distritos, existen grandes desequilibrios entre la oferta y la demanda del servicio. Las mayores diferencias en cuanto a la cantidad de metros recorridos a la línea más próxima se dan en los estratos de población con menores recursos. Dicha situación genera condiciones de desigualdad e inequidad afectando la calidad de vida de la población. 
El desarrollo de las futuras políticas públicas debe orientarse a garantizar la obligatoriedad del servicio público en aquellas zonas de la ciudad donde el TPP tiene actualmente un bajo nivel de disponibilidad.

Asimismo, se pretende destacar la necesidad de considerar el territorio como elemento fundamental en la elaboración de las políticas públicas. El servicio público de Transporte Urbano de Pasajeros debe ser pensado por el Estado desde el territorio a nivel ciudad o a nivel metropolitano.

Se concluye que la política pública sectorial del TPP debe ser pensada desde el territorio buscando adaptar el servicio a las necesidades de la población a los fines de reducir los signos de inequidad que se observan, principalmente, en aquellos sectores de la población que menos recursos poseen y que se encuentran cautivos de este medio de transporte.

\section{NOTAS}

1 Estos sistemas permiten combinar la información numérica (datos obtenidos del Censo Nacional 2010) con la información geográfica (mapas digitales de la ciudad a nivel de radiofracción). A su vez, la realización de los cálculos estadísticos para la obtención de los resultados. Es una herramienta utilizada para determinar áreas de emergencias.

\section{PARA CITAR ESTE ARTÍCULO:}

Giménez Puentes, M.P. (2017). "Recensión de tesis: “El Transporte Público de Pasajeros en la ciudad de Santa Fe: un análisis de accesibilidad y vulnerabilidad social sobre el territorio" ", DAAPGE, año 17, № 29 (jul-dic), 2017, pp. 131-159. Santa Fe, Argentina: UNL. 